\title{
Setup of a Beam Control System for High Power Laser Systems at DLR
}

\author{
Ivo Buske, Andreas Walther \\ Deutsches Zentrum für Luft- und Raumfahrt, Pfaffenwaldring 38-40, 70569 Stuttgart, Germany, \\ Ivo.Buske@dlr.de
}

\begin{abstract}
Different types of high power or high energy lasers in the multi $\mathrm{kW}$ class are currently available or are under development with promising progress reports. A major challenge is to deliver as much as possible of the available power onto a small and fast moving target over a long distance through a disturbing atmosphere.

High resolution imaging is a common way to identify the category of targets dedication and to determine the spatial position relative to the observer. By illuminating the target with a laser the imaging system becomes more resilient towards ambient light and the exposure time can be reduced drastically. Fast and deterministic control loops are demanding for the moving parts in order to maintain a high accuracy for the pointing of the turret and aiming of the laser countermeasure system.

Here, we report on the progress of such a beam control system developed at the Institute of Technical Physics of DLR. In an overview we present the beam control system and explain different sub-systems. Performance tests were taken at our outdoor test range. We investigated various scenarios for probing the limits of the tracking and pointing accuracy with a target sample mounted on a fast moving linear stage. We present first results of the beam control system performance.
\end{abstract}

Keywords: laser beam control, laser tracking, high power laser, directed energy

\section{INTRODUCTION}

High Energy Laser (HEL) weapons have unique attributes which distinguish them from limitations of kinetic energy weapons. Its inherent precision strike capability and ability to engage while looking at the target are the most distinguishing attributes [1]. Today different types of high power or high energy lasers in the multi $\mathrm{kW}$ class are currently available or are under development with promising progress reports [2]. The next step is to transfer the laser power from the source to the target. More or less this beam control system has to focus as much laser power as possible on a small spot on a fast moving target far away through the atmosphere. This cannot be achieved by using a conventional beam director. Additionally different add-on systems have to work together to satisfy the requirements.

Often complex system functionality can be split in sub-tasks. This allows a detailed investigation and optimization of specific sub-systems. To evaluate the performance of such sub-systems it is mostly not necessary to test the system in the field of their application. Quite the contrary a dedicated test environment can work out the influence of specific environment conditions which are normally superimposed. Environment parameters like weather conditions, system base vibrations or target characteristics can be measured and recorded in a detailed protocol to find out the interfering effect on the sub-system. A determined way to optimize a sub-system is to fix single parameters by turning off their influence and observe the residual system characteristics.

At DLR the Institute of Technical Physics built such a unique test environment. A dedicated laser test range is available to evaluate beam control systems. Propagations of high power or high energy lasers are analyzed under safety conditions. An outstanding agile target platform enables the use of a variety of moving targets. Thereby the position of the target is known with high accuracy for static as well as dynamic operation. The moving platform provides the testbed for all motion relevant investigations. All beam control sub-systems are tested on laser test range. Under this unique test environment we are able the record weather and optical turbulence data to identify their influence on the beam control performance. Cooperative and uncooperative targets are available to determine the influence of signal quality on the system performance. 


\section{BEAM CONTROL EVALUATION ENVIRONMENT}

\subsection{DLR laser test range}

To evaluate beam control systems under outdoor conditions a laser test range is set-up at DLR test site Lampoldshausen (Fihure 1). Two buildings are installed to contain the beam control system on the one side and the evaluation equipment on the other side. In the transmitter station (TS) also high power and high energy lasers are located. On the opposite side in the receiver station (RS) an agile target platform is operated clamped with different target samples and a variety of measurement equipment to characterize laser beams. The propagation range has a distance of $130 \mathrm{~m}$. The standard propagation height is $1 \mathrm{~m}$ above asphalt ground. For laser safety reasons this height is limited and admittance is strictly controlled. The restricted area is supervised from a remote station.

Different kinds of detectors monitor the environment to support the laser beam propagation experiments. Only to mention some scintillometer, solar radiation, visibility monitor, air and asphalt temperature, wind speed and wind direction. Often an exact statement about the weather conditions is prerequisite to evaluate experimental results. Detectors operate continuously night and day. They are recorded independent of other activities. All measurements are collected 24/7 at different atmospheric conditions like rain, fog, haze, snow, dust, sunshine. Since 2008 all measurements are statistical analyzed. That allows precise predictions for just running experiments.
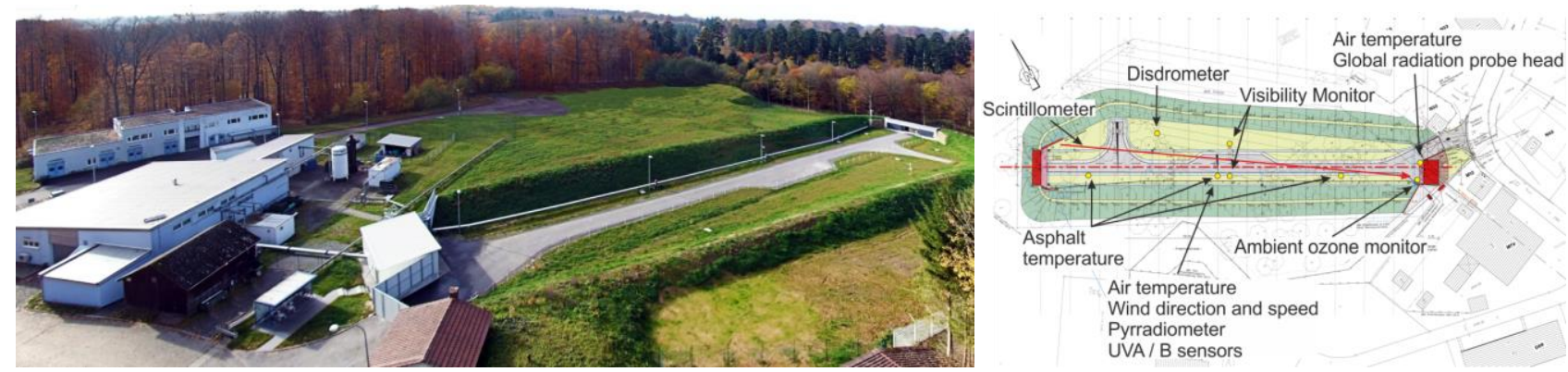

Figure 1. Left: Aerial photography of DLR laser test site in Lampoldshausen with transmitter station, test range and receiver station. Right: Different types of environment measurement systems and their position to monitor selected parameters $24 / 7$.

\subsection{Agile target platform and intelligent targets}

Beam control systems are generally used to measure the position of fast flying targets. Furthermore the beam control system should track the target with high precision. That qualifies the system to point a laser beam onto the target. To evaluate the beam control system performance it is a basic requirement to know the exact position of the target. Therefore an agile target platform (ATP) was developed and installed into the receiver station (figure 2). The linear translation stage with a stage length of $1.7 \mathrm{~m}$ was tightened on a heavy granite base ( 3 to) to avoid motion relative to the ground during acceleration phases. Direct drives with high torque motors are integrated for high acceleration, yielding an outperforming agility of the stage. Position signals are supplied by optical high resolution encoders. Additionally, the current position can be read off from a position synchronous output (PSO) pin. Thermometers are used to monitor the temperature during the experiment for calculating their influence on position accuracy. External calibration systems like laser distance sensor or high speed laser interferometer review the system accuracy in a regular manner. All these features cause a high dynamic precision of the platform. As an example for a target mass of $5 \mathrm{~kg}$ an acceleration of $6 \mathrm{~g}$ can be applied to achieve a homogenous velocity of $5 \mathrm{~m} / \mathrm{s}$ over $1 \mathrm{~m}$ central travel distance. The dynamic position accuracy for $5 \mathrm{~m} / \mathrm{s}$ was determined to be better than $10 \mu \mathrm{m}$. The position repeatability was measured from different motion profiles. In all motion profiles the maximum deviation from the required positon was $<1.5 \mu$ m RMS. Smallest step size is $0.1 \mu \mathrm{m}$. The velocity stability at $5 \mathrm{~m} / \mathrm{s}$ is down to $+/-10 \mathrm{~mm} / \mathrm{s}$.

To protect the agile target platform a transparent cage was installed. Due to the location nearby a forest animals have to be keep away from the system. The cage is pressurized by nitrogen to minimize pollution by dust and pollen. It covers the stage also for safety reasons.

Different kinds of targets can be mounted on the agile target platform. The targets are replaceable in a convenient way. All power support or signal data cables are integrated in a cable drag chain. The background is normally black or white. 
The observer has the possibility to control and monitor all experiments over IP cams. Synchronized data acquisition using the IEEE 1588 standard is available between the transmitter and receiver station. A time jitter of $120 \mathrm{~ns}$ RMS was proved.
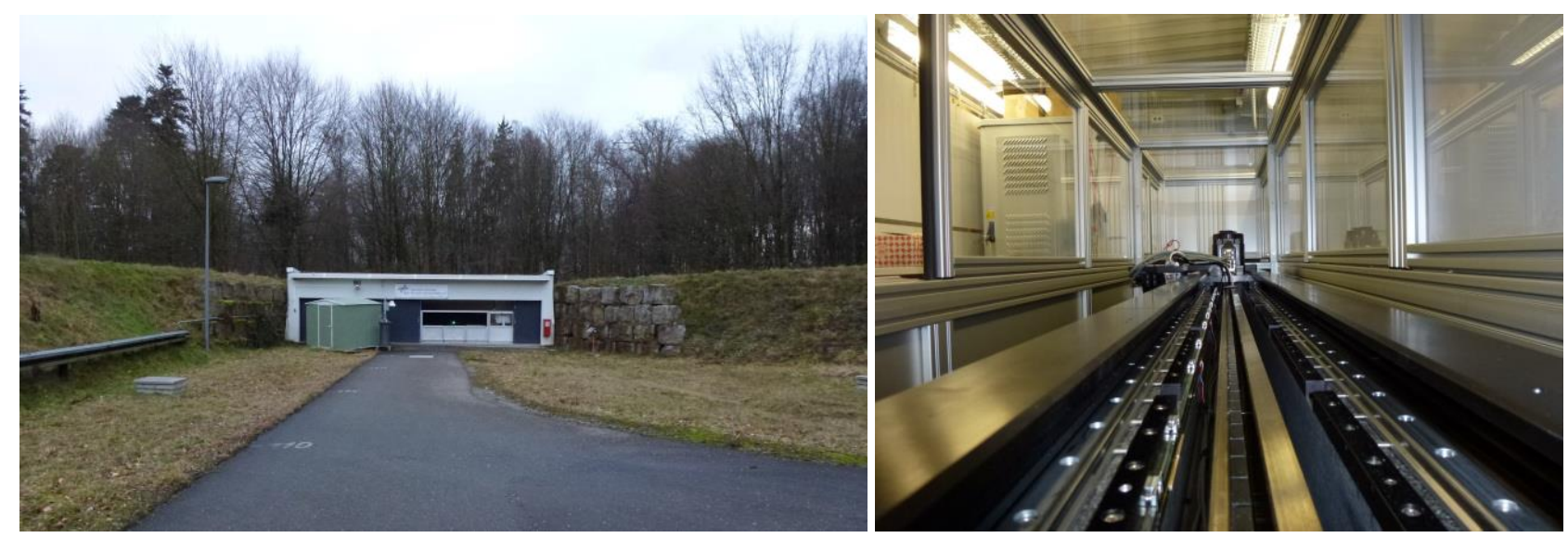

Figure 2. Left: View of the receiver station with open gate. Cage of the agile target platform is open in transmitter direction. Right: Sliding carriage system of agile target platform within the cage. A traveling distance of $1.7 \mathrm{~m}$ can be used.

\section{LASER BEAM CONTROL SYSTEM}

Splitting beam control systems in sub-systems offers the possibility to optimize and evaluate well-defined task. Typical sub-systems can be found mostly in every laser beam control system (figure 3). First a target illumination laser which can be used as an extra ordinary flash lamp. The laser provides the required number of photons to exposure the camera focal plane array within sub-ms. It generates glint reflexions and is the source for laser ranging or gated viewing applications. Second an optical position measurement system is required to determine the position of the target relative to the observer site. High resolution imaging permits the identification of the target. The distance between the target and the observer can be measured by laser ranging. The position information is required as an input for an optical tracking system, which follows the selected target and orientates the measurement system in the line of sight by actuators. All these sub-systems have to be operated correctly before the glint tracker can be turned on. Glint tracker ensures the high dynamic alignment of remote or countermeasure laser power on the most vulnerable target structure. Although each subsystem is assigned for a dedicated task the whole functionality can only be fulfilled if all sub-systems are working perfectly together.

\section{beam control system}

\section{Passive Optical Tracker CTS (Coarse Tracking System)}

FTS (Fine Tracking System)

TILL (target illumination laser)

Active Optical Tracke GTS (Glint Tracking System) laser test range

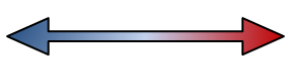

ATP (Agile Target Platform)

IT (Intelligent Target)

Figure 3. Schematic overview about the overall system required for development and evaluation the beam control system. Besides the beam control system also the evaluation system can be split in reasonable sub-systems. 


\subsection{Mobile Optical Tracking System (MOTSy)}

The Mobile Optical Tracking System (MOTSy) is one of the core components of our beam control system (figure 4). It is used to determine accurately the relative position of the target to the optical axis of the laser beam transmitter. Additionally the high resolution optics allows the identification of the target and the determination of the glint position relative to the target structure. Distance information setting up focus adjustment of the high power laser. Time Space Position Information (TSPI) delivers important data to decision makers. Stabilized images of the region of interest identify the threat under engagement.

Optical Tracking Systems process the target position information to align the position measurement system, assuring target is hold within the field of view of the detector. The system has to be agile to follow the target. It includes a highly accurate position encoder to support the position measurement system. Inertia of the system tends to result in deviation of the aligned optical axis to the target position. A woofer tweeter setup improves the tracking accuracy by implementing an agile beam steering system on the tracking platform.

MOTSy is intended as a research platform for different applications. Its unique properties results from several improvements and optimizations. Primarily MOTSy consist of a direct motorized astronomical mount. The advantages of such a mount are the precise angle position measurement together with an excellent cost-value ratio. Normally established in equatorial mount setup we arranged the system in altitude-azimuth configuration. The extension arm is rigidly constructed to clamp all sensors, cameras and objectives on it without any mechanical deformations. A maximum load of $65 \mathrm{~kg}$ can be installed. The mount is fully controlled by USB channel. A direct encoder output was created to measure the angular position with an accuracy of 0.02 " just in time. That enables the synchronization the image exposure with the current angular position of the mount. MOTSy was built in a mobile version to have full flexibility within the operational area. MOTSy can be easily mounted either on rigid post or movable vehicles. We improved the limits of the astronomical mount by adjusting the initialization parameters. Therefore we obtain a maximum velocity of $25 \%$ s and an acceleration of $50^{\circ} / \mathrm{s}^{2}$.

MOTSy includes two different camera systems. A wide field zoom camera with $6 \mathrm{MP}$ and a frame rate of $20 \mathrm{~Hz}$ generates an overview about the scenery. Within the field of view of $450 \mathrm{mrad}$ every subject can be marked by the operator. Tracking algorithm directs the mount to the user selected target. The second camera systems consist of a $800 \mathrm{~mm}$ F 5.6 tele objective. Focus and iris aperture can be controlled by RS232 interface. The CMOS 2MP camera creates a closer field of view of $7 \mathrm{mrad}$. Depending on the region of interest (ROI) the frame rate increase from $85 \mathrm{~Hz}$ at full resolution to over $1 \mathrm{kHz}$ at $100 \times 100$ pixels.

To evaluate the measurement accuracy we assemble MOTSy in an indoor location. This will prevent distortions which will be occurring due to atmospheric turbulence. The position measurement accuracy of moving target was determined to be better than $2 \mu \mathrm{rad}$. Repeating the trials on the laser test range we verify a position measurement accuracy of around $10 \mu \mathrm{rad}$. This value is mainly dominated by atmospheric turbulence which induced additionally image jitter. To ensure a sufficient signal to noise ratio within the short exposure time the target consists of a bight LED source [3].
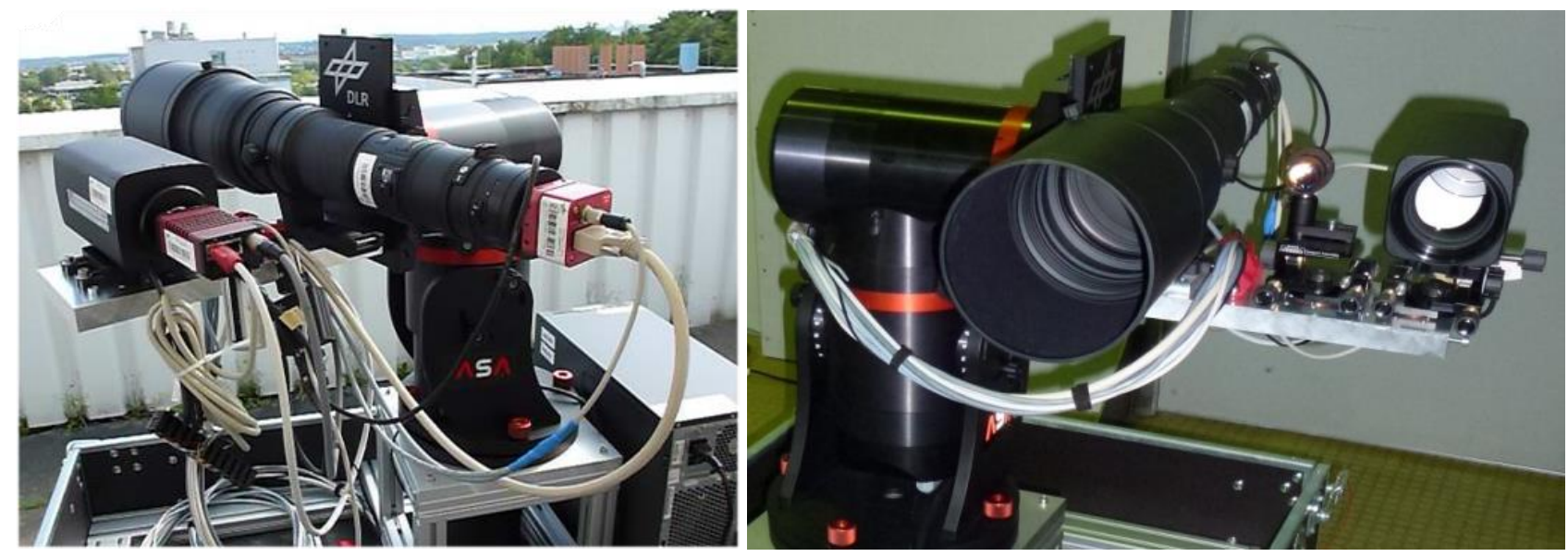

Figure 4. Both pictures show the main components of the mobile optical tracking system (MOTSy). The system consists of different camera systems and an optimized astronomical mount. Here the mount was installed on a transportable vehicle. 


\subsection{Target Illumination Laser (TILL)}

A target illumination laser system fulfills many different tasks. First it can be used for illuminating the scenery to decrease the required exposure times of observing cameras. The incoming light can be filtered to the wavelength of the illuminator. Therefore ambient natural or artificial light will be suppressed in the picture. Second the illumination creates bright glint reflexes on the target surface. A glint tracker requires these spots. High precision and timed laser pulses are prerequisite to use active imaging or gated viewing methods, enabling the suppression of light coming from the background or from scattered particle located between the laser system and the target. Image contrast can be further improved. At last laser pulses can also be used to determine the distance to the target by analyzing the time-of-flight measurements.

We establish a diode pumped solid state laser (DPSSL) for target illumination (figure 5). The laser system contains two laser output options which are both collinear and collimated. The laser transmitter emits laser energy of $50 \mathrm{~mJ}$ at $1064 \mathrm{~nm}$ or $25 \mathrm{~mJ}$ at $532 \mathrm{~nm}$. The transmitted wavelength can be changed by a motorized mirror. Typical laser parameters are the pulse length of $8 \mathrm{~ns}$ and the pulse repetition rate of $1 \mathrm{kHz}$. The laser is distinguished by its beam quality of $\mathrm{M}^{2}<1.4$. Beam parameters can be monitored continuously in a separated beam line using a sampled beam copy. Following laser beam parameters can be recorded: beam profile, beam pointing stability, beam collinearity, pulse shape, pulse length, trigger jitter.
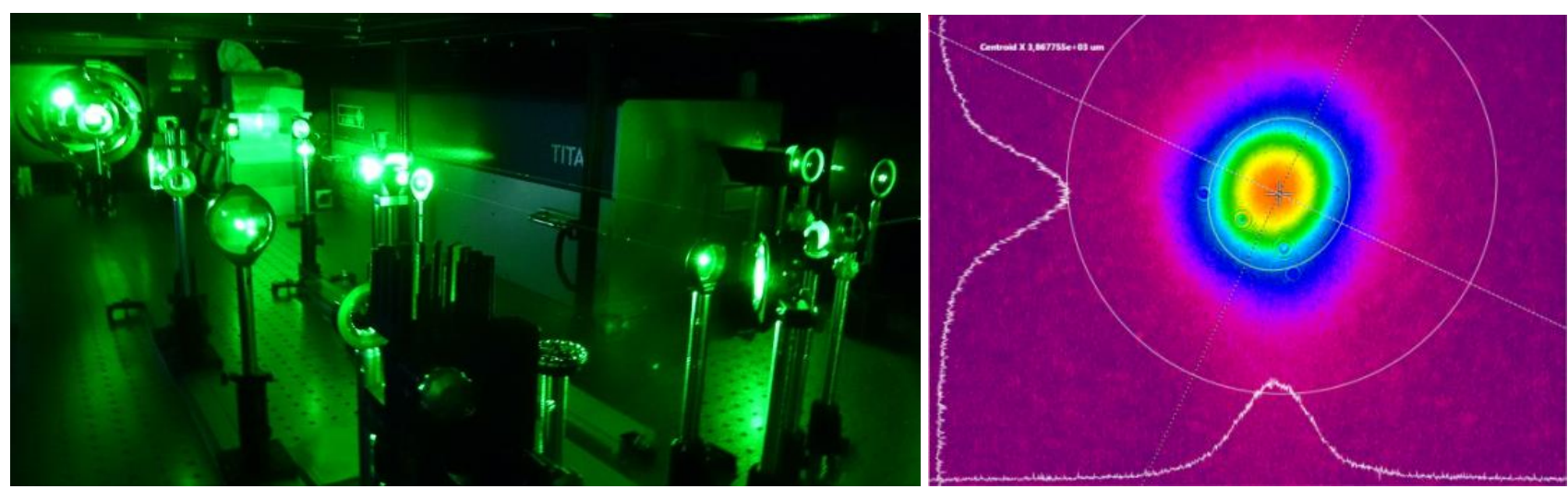

Figure 5. Left: Beam path of the target illumination laser. Different beam shaping functions are implemented to adapt the beam profile at the target zone. Right: Excellent infrared beam profile of the laser system during operation with $\mathrm{M}^{2}=1.1$.

The laser output is separated from the high power laser output and from the optical tracking system. It has to be ensure, that the beam profile illuminates the desired area at the target zone. A beam steering mirror facilitates the autotracking of the illumination cone. The optical design of the TILL beam shaping was calculated separately for the vertical and horizontal plane. That allows the possibility to achieve an aspect ratio of the illumination zone. Likewise, the illumination diameter in the target zone can be matched by adjusting collimation optics in the beam path. To distinguish the illumination from the cage of the agile target platform an adjustable aperture was inserted in the beam path. The plane of the aperture was imaged on the target plane to achieve a nearly top hat illumination profile.

\subsection{Glint Tracker System}

A Glint Tracker System (GTS) is a high speed control loop tracking sub-system. Its main task is the compensation of external induced mechanical vibrations and atmospheric induced tip/ tilt beam jitter. These distortions occur usually at frequencies where servo controlled actuators can't follow. High speed galvo or piezo actuators are predestined solutions.

Typically glint tracker systems require a bright light source on the target. A target illumination laser can generate such a glint on the target surface. Optics imaged the laser glint onto a high speed position sensor. Thereafter the glint position will be measured and the actuating signal will be calculated. Follow up by a high speed beam steering actuator, which corrected the misaligned beam direction. The performance of the control loop defines how accurate the glint of the target can be followed by the glint tracker. As well as the glint tracker beam path is collinear aligned to the high power laser beam path. Due to the optical setup the beam pointing of the impact laser system will be simultaneously aligned to the target. Consequently the high power or high energy of the laser beam will be concentrated on the glint position on the target. To select the most vulnerable point on the target it has to be determined the correct position offset to the glint 
position. This task can be executed by the optical tracking system (MOTSy). Its high resolution imaging system is well suited to identify the glint positon relative to the target shape.

In figure 6 the schematic setup of the glint tracker beam path is shown. The target illumination laser creates a glint reflexion. This incoming light is reflected by the 12" tracking tip/tilt mirror. The folding mirror provide target tracking capabilities across wide field area. The field of view on the laser test range is limit to $+/-10 \mathrm{mrad}$. A telescope consist of a 12" off axis mirror, a folding flat mirror and an achromate implement a magnification of the beam by a factor of 10 . A fast tip/tilt mirror is located near the back focal length of the achromate. It is used to compensate the glint position within an optical range of $+/-4 \mathrm{mrad}$. A laser processing head includes a beam divider which splits the visible light coming from the target glint and the infrared transmitted high power laser beam. Additional filter ensure that infrared light is blocked in sensor beam path. A lens imaged the target glint onto the glint sensor. Currently we use for convenient reasons a USB3 cmos camera as the glint sensor. The flexibility of a focal plane array offers an advantage during the development process. Camera parameter like ROI can be adapted in an easy way. On the contrary a limiting factor like data bandwidth could be an issue. Additionally image processing can be very time consuming. Experiments proof that all time constrains will be achieved. Focusing is realized by adjusting the distance between lens and glint sensor. All optic elements are dielectric coated with a high reflection coating for $532 \mathrm{~nm}$ and $1030 \mathrm{~nm}$ wavelength for high power laser applications. The high power beam path is collinear arranged and propagate in the opposite way through the optics. The high power laser source is fiber coupled into the laser processing head.

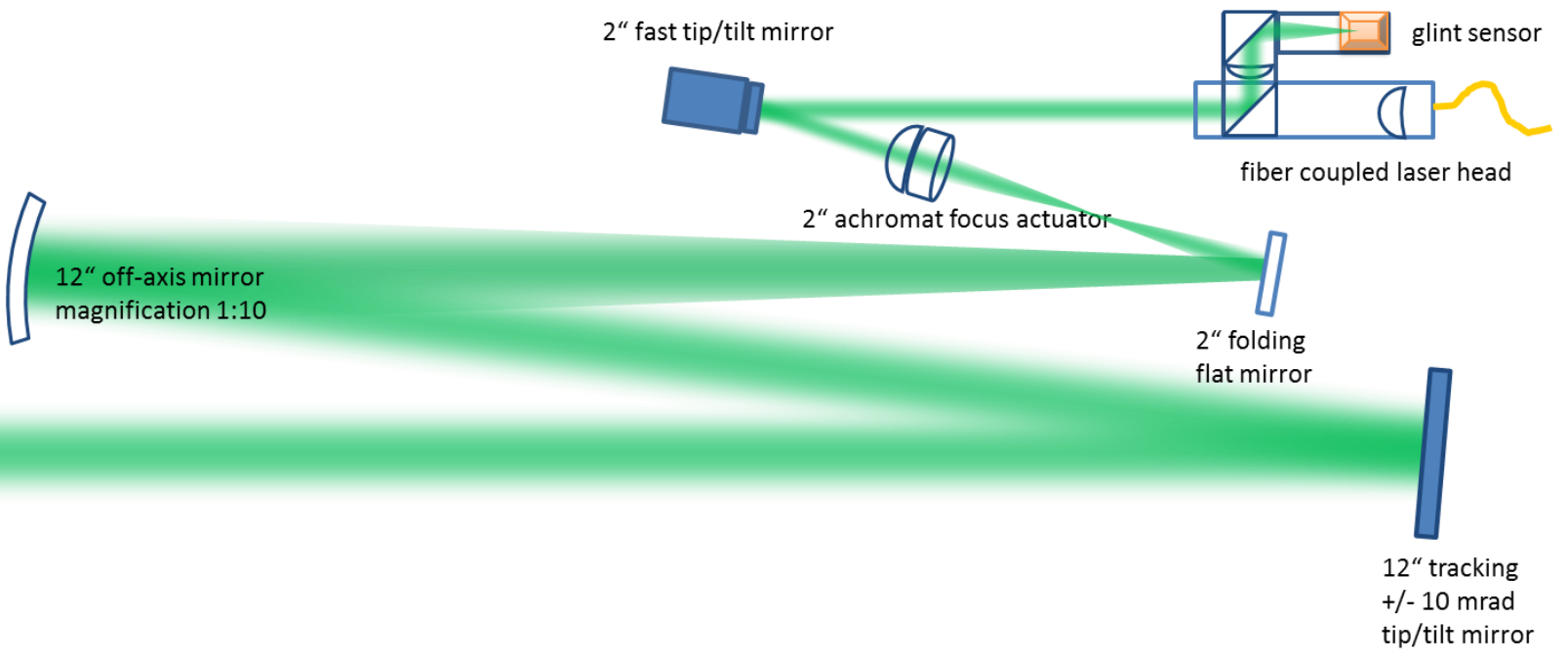

Figure 6. Beam path of the glint tracker system which is collinear arranged with the transmitted high power laser. The glint will be imaged on a glint sensor. Optic adapt the beam diameter by a telescope. Steering mirrors are included to track the glint reflex.

The custom design of the glint tracking actuator is a piezo driven tip / tilt 2" flat mirror (figure 7). Highlights are the thermal isolated and replaceable mirror holder. The heat loaded by the high power laser is shielded from the heatsensitive piezoelectric materials. Mirror substrate and holder mass are reduced for low inertia. Thermal monitoring serves as a warning sensor. External connected air cooling was realized by external tubes to prevent atmospherical turbulence within the beam path. All components are sealed against environmental pollution. A rigid base was installed to preserve the mirror mechanical system resonance of $>1 \mathrm{kHz}$ for the whole system. We evaluate the piezo tip/tilt mirror and obtain for a mechanical deflection angle of $+/-2 \mathrm{mrad}$ a max. sinusoidal frequency of $150 \mathrm{~Hz}$. At $300 \mathrm{~Hz}$ oscillation we measured a deflection of $+/-1 \mathrm{mrad}$. Test under high laser power load (5 $\mathrm{kW})$ are carried out to demonstrate full functionality over long operation period.

Recently, investigations were done to characterize the performance of the glint tracker system for compensating atmospheric turbulence induced beam jitter. Therefore the target was fixed in a static position. The glint from the target was simulated by a fiber coupled LED source. This ensures a perfect point source with constant light output. The variance of the angle of arrival on the entrance aperture creates an image jitter in the focal plane. The point source was imaged onto the focal plane array with the dimension of 32 × 32 pixels. The control closed loop could be run with frame rates of $2.3 \mathrm{kHz}$. The image processing calculates the centroid of the image spot in time. All results are recorded in a log 
file. Data analyzes shows a displacement of the spot centroid with a standard deviation between 5 up to $20 \mu$ rad RMS. The beam jitter strength depends on the varying value of the refractive index structure coefficient $c_{n}{ }^{2}$. Different environment parameters have an impact on $\mathrm{c}_{\mathrm{n}}{ }^{2}$. Besides air temperature and wind speed also the beam propagation height over ground have significant influence on the strength of $c_{n}^{2}$.

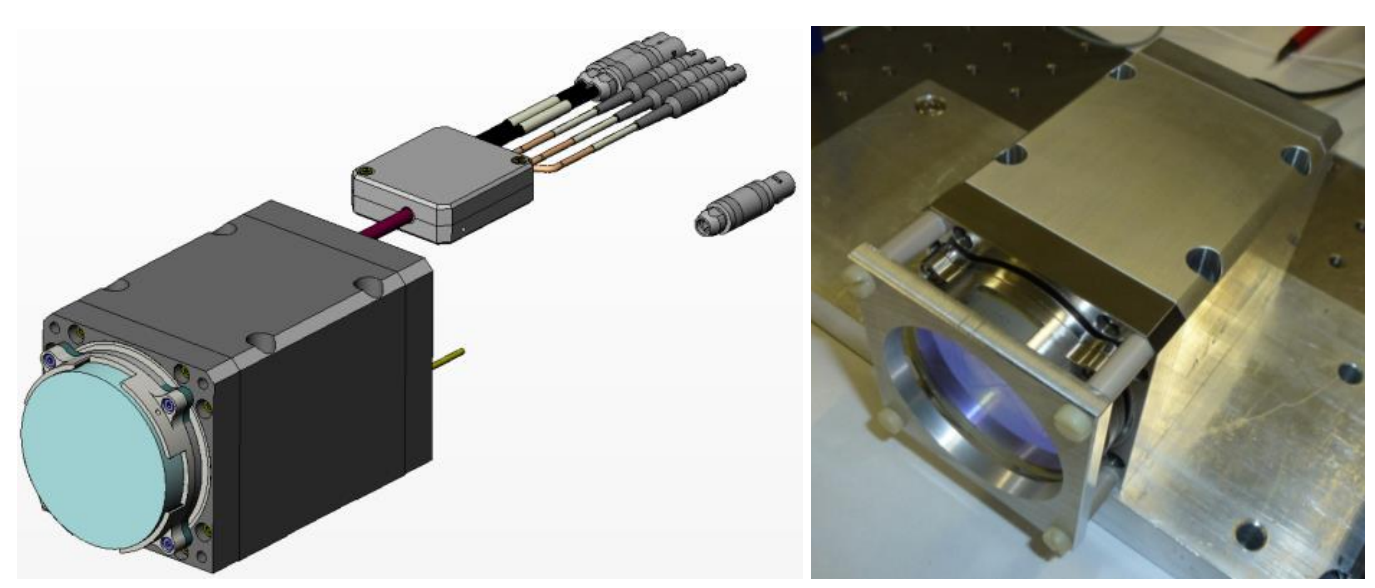

Figure 7. Sketch and photography of the piezo tip / tilt mirror system. Improvements due to the custom design enable applications like high power laser systems.

Closing the loop by turning the glint tracker system on the piezo tip / tilt mirror are controlled to compensate for the beam jitter. Measurements shows that the standard deviation will be reduced to $1 . .5 \mu$ rad RMS. This corresponds to an improvement by a factor of 5 . Figure 8 shows the temporal characteristics of the vertical position signal and mirror control voltage during open loop and closed loop operation.

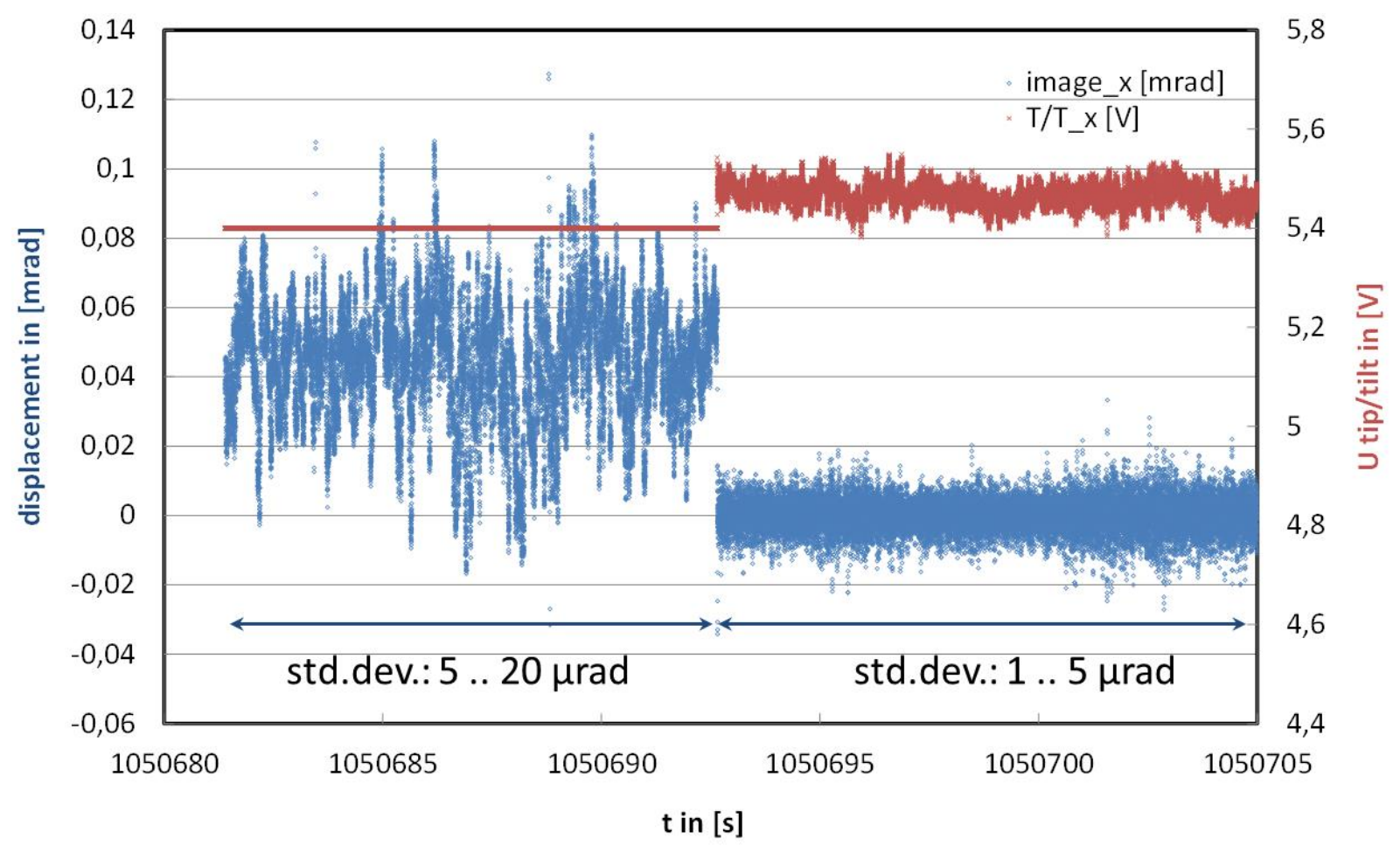

Figure 8. Beam jitter displacement induced by natural atmospherical turbulence before and after activating the glint tracker system. 


\section{SUMMARY}

We reported in this paper the experimental setup of a beam control system for high power laser systems. Due to their specific task different sub-systems were defined. Indoor and outdoor experiments were carried out to evaluate the performance of each sub-system.

A mobile optical tracking system (MOTSy) was developed as a sub-system to measure the position of the targets and track their trails. We demonstrate indoor a position measurement accuracy of $<2 \mu \mathrm{rad}$. Outdoor we achieve an accuracy $<10 \mu \mathrm{rad}$ because the influence of atmospheric turbulence cannot be neglected.

A glint tracking system consists of high frame rate glint sensor and piezo tip/ tilt mirror for high power applications. Due to the collinear beam path this sub-system also improves the beam pointing stability of the transmitted high power laser. The glint tracker is able to compensate external vibrations and atmospheric turbulence between 5 .. $20 \mu$ rad by a factor of 5. A dedicated laser test range was used to evaluate the performance also in outdoor condition and to work out the influence of specific environment conditions.

\section{REFERENCES}

[1] Cook, "High-energy laser weapons since the early 1960s," Opt. Eng. 2, (2013).

[2] Extance, " Military technology: Laser weapons get real," Nature 521, (2015).

[3] Walther, Buske, "Accuracy analysis of a mobile tracking system for angular position determination of flying targets," Proc. SPIE 9989 (2016). 